\title{
An Integrated Framework of Drivetrain Degradation Assessment and Fault Localization for Offshore Wind Turbines
}

\author{
Wenyu Zhao ${ }^{1}$, David Siegel ${ }^{1}$, Jay Lee ${ }^{1}$, and Liying $\mathrm{Su}^{2}$ \\ ${ }^{1}$ NSF I/UCRC for Intelligent Maintenance Systems, University of Cincinnati, Cincinnati, Ohio, 45221-0072, USA \\ zhaowy@mail.uc.edu \\ siegeldn@mail.uc.edu \\ jay.lee@uc.edu \\ ${ }^{2}$ Sinovel Wind Group Co., Ltd., Beijing, 100872, China \\ suliyingamily@163.com
}

\begin{abstract}
As wind energy proliferates in onshore and offshore applications, it has become significantly important to predict wind turbine downtime and maintain operation uptime to ensure maximal yield. Two types of data systems have been widely adopted for monitoring turbine health condition: supervisory control and data acquisition (SCADA) and condition monitoring system (CMS). Provided that research and development have focused on advancing analytical techniques based on these systems independently, an intelligent model that associates information from both systems is necessary and beneficial. In this paper, a systematic framework is designed to integrate CMS and SCADA data and assess drivetrain degradation over its lifecycle. Information reference and advanced feature extraction techniques are employed to procure heterogeneous health indicators. A pattern recognition algorithm is used to model baseline behavior and measure deviation of current behavior, where a Self-organizing Map (SOM) and minimum quantization error (MQE) method is selected to achieve degradation assessment. Eventually, the computation and ranking of component contribution to the detected degradation offers component-level fault localization. When validated and automated by various applications, the approach is able to incorporate diverse data resources and output actionable information to advise predictive maintenance with precise fault information. The approach is validated on a $3 \mathrm{MW}$ offshore turbine, where an incipient fault is detected well before existing system shuts down the unit. A radar chart is used to illustrate the fault localization result.
\end{abstract}

\footnotetext{
Wenyu Zhao et al. This is an open-access article distributed under the terms of the Creative Commons Attribution 3.0 United States License, which permits unrestricted use, distribution, and reproduction in any medium, provided the original author and source are credited.
}

\section{INTRODUCTION}

With the rapid increase in the adoption of wind power for renewable energy generation, wind farm development and wind capacity installation have seen extensive growth. As Global Wind Energy Council (2012) pointed out, global capacity has reached $237 \mathrm{GW}$ in 2011 and is projected to achieve $759 \mathrm{GW}$, which is more than three times current capacity, by the year of 2020 .

Meadows (2011) shows that a 1975 MW offshore capacity has been installed in Europe, whereas a $135 \mathrm{MW}$ capacity is available in China; for the United States, the forecast of offshore capacity is $10 \mathrm{GW}$ by 2020 and $54 \mathrm{GW}$ by 2030 . However, availability and reliability of offshore turbines are imposing challenges for productive and efficient offshore wind farms.

A comprehensive report by National Renewable Energy Laboratory (2010) provided similar insight that: U.S. offshore wind power has great potential of supporting a considerable percentage of electricity needs; while the improvement of reliability through condition monitoring is one of major technology trends that will greatly support operations and maintenance for turbines both onshore and offshore.

CMS has been an emerging technology for monitoring turbine health status and diagnosing component failures. A study by LeBlanc and Graves (2011) shows that, the application of CMS is rising despite initial doubt of its capability. In certain offshore wind farm guidelines, CMS is even mandatory for turbine monitoring (GL Renewables Certification, 2012). A framework of CMS is provided in the study as well, where typical requirements of sensor locations are shown. The benefit of adopting CMS is discussed and justified, based on failure rates of key components and related cost. It proves that, on average, 
predicting one gearbox failure can clearly justify the budget of deploying a CMS on the turbine system. In addition, McMilan and Ault (2007) investigated Markov model between drivetrain components and quantified revenue and risk of condition monitoring, based on reliability data of the components.

As detailed by ISET (2005), CMS utilizes various types of communication infrastructure to transfer real time sensor data and control information to data centers, where servers are used to host and process the data. Another study (Amirat, Benbouzid, Al-Ahmar, Bensaker and Turri, 2009) also provided a review of data collection schemes for the electrical system, blade and drivetrain condition monitoring.

In literature, much research is being conducted for condition monitoring of wind turbines based on data infrastructure. $\mathrm{Lu}, \mathrm{Li}, \mathrm{Wu}$ and Yang (2009) gave a diagnosis review of the gearbox, bearing generator, power electronics, rotor, blades and overall system with condition monitoring techniques including vibration, torque, oil debris, temperature, acoustic emission and electric current \& power analyses. Hameed, Hong, Cho, Ahn and Song (2009) provided a related review of fault detection methods for global and subsystem levels based on CMS. Crabtree, Feng and Tavner (2010) developed a multivariate approach that combines vibration and oil debris analysis for detecting gearbox failure at an early stage. Entezami (2010) proposed an overview and approach to connect the control system with turbine condition monitoring. Sheng and Veers (2011) described the gearbox reliability collaborative research at the National Renewable Energy Laboratory, where a fully instrumented drivetrain test bed is built for generating lab test data.

Furthermore, SCADA system is also frequently used for monitoring wind turbine condition. Commonly used variables in different SCADA systems are shown in Table 1.

\begin{tabular}{l|l}
\hline Category & Variable Examples \\
\hline Ambient & Temperature, wind direction, wind speed \\
\hline Blades & Pitch angle \\
\hline Controller & Hub temperature, Ground temperature. \\
\hline Gear & Gear bearing temperature, oil temperature \\
\hline Generator & Bearing temperature, rotation speed \\
\hline Grid & Production voltage, current, power factor \\
\hline Hydraulic & Hydraulic oil temperature \\
\hline Nacelle & Direction, temperature \\
\hline Production & Average power, accumulated power \\
\hline Rotor & Rotation speed \\
\hline System & Logs of active alarms, turbine state \\
\hline $\begin{array}{l}\text { Hour } \\
\text { Counter }\end{array}$ & Service hours \\
\hline
\end{tabular}

Table 1. Commonly Used SCADA Variables
In a study by Qiu, Feng, Tavner, Richardson, Erdos and Chen (2012), SCADA data from an onshore turbine is used for alarm analysis and probability-based reliability modeling. SCADA data is also suitable for evaluating turbine power generation performance, which is complicated by the dynamic environment parameters and operation conditions (Lapira, Siegel, Zhao, Brisset, $\mathrm{Su}$, Wang, AbuAli and Lee, 2011).

In most of the available literature, CMS and SCADA systems are used separately for condition monitoring purposes, mainly due to the issue of data availability in certain research activities. Moreover, the majority of tools and techniques are developed and validated on lab-scale test beds. To address such issues, a degradation assessment framework is proposed to integrate CMS data and SCADA variables for the evaluation of drivetrain degradation. Although usually used for a different purpose than a CMS, SCADA provides operational information that can assist the screening and processing of CMS data. In addition, some SCADA systems can provide variables that can serve as health indicators of drivetrain components. Previous research, including Qiue et al. (2009) and Edwin, Theo, Henk, Luc, Xiang and Simon (2008), show that SCADA variables can be used for fault detection at early stage, especially through analyzing temperature measurement from drivetrain components. The framework is eventually validated with an offshore turbine drivetrain.

The remainder of the paper is organized as following: Section 2 describes the methodology of integrating SCADA system data and CMS data, extracting and selecting features, assessing drivetrain degradation and identifying fault location; Section 3 demonstrates an application of the methodology in monitoring the drivetrain for a $3 \mathrm{MW}$ offshore turbine, as well as a monitoring platform prototype with visualization tools; Section 4 discusses the conclusion of presented work, and plan for future development and validation; acknowledgement and references ensue as the last portion of paper.

\section{Methodology}

The overall framework integrates selected information from both SCADA and CMS systems, provides global degradation assessment of the drivetrain, and identifies faulty component(s) when fault detection is determined to be positive. The systematic methodology is shown in Figure 1 .

SCADA variables that are related with wind turbine operation are initially used to assist in deciding if individual CMS data instances can represent the true degradation condition for drivetrain. CMS data from all sensors in retained instances are then processed by a set of feature extraction tools, while SCADA variables that indicate drivetrain conditions are selected. CMS features and 
SCADA variables are then concatenated and input to a degradation assessment method, to evaluate how the overall condition differs from a baseline. When degradation is significant and fault detection is confirmed, the location of the fault is decided based on each component's contribution to the overall degradation. Eventually, the analytical results are visually presented.

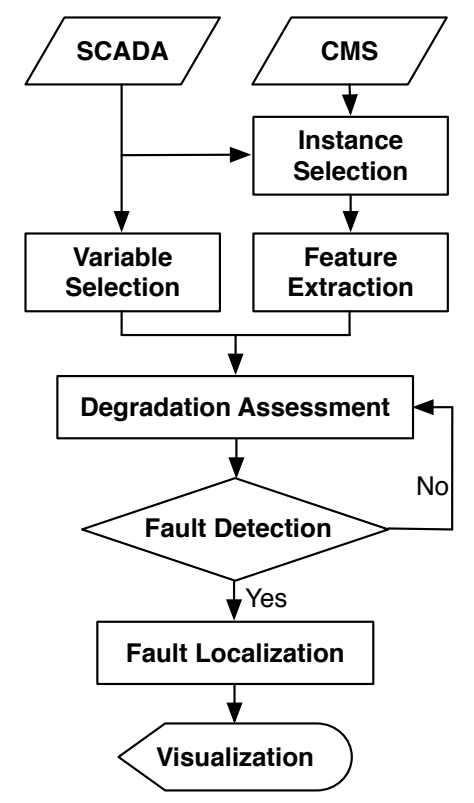

Figure 1. The integrated framework for drivetrain degradation assessment and fault localization

The rationale and techniques for each step are described as follows.

\subsection{CMS Instance Selection}

In applications of condition monitoring systems for wind turbines, it is a common practice to configure the sampling scheme as a routine program, so that several seconds or minutes worth of vibration waveforms with high sampling frequency from all instrumented sensors are acquired and stored at certain time instances throughout a day. This strategy is due to the limitation of proper infrastructure for data acquisition and transferring, and the concern of computational capacity for large-scale wind farms. In a few cases, vibration data is processed and only its features will be archived for further investigation; nevertheless the feature extraction is usually still time-based with a static period.

For a condition monitoring system that utilizes multiple accelerometers to measure the vibratory behavior of the drivetrain, it is important to decipher the quality of vibration data before actually processing the data. If the instance of data waveforms does not characterize the drivetrain's true health at the time of acquisition, including such instance in later analysis will generate false health information and affect decision making for maintenance. With rule-based criteria learned from wind farm operation and the control mechanism, irrelevant CMS data instances should be discarded based on SCADA measurements and only meaningful instances are kept for subsequent analysis. For example, if it is detected that the rotor speed has been zero for certain duration and there has been no rotation for the drivetrain, CMS data instance collected within this duration is determined to not contain vibration information that can be used for degradation assessment. Such instances should be rejected prior to further analysis.

\subsection{SCADA Variable Selection}

For majority of SCADA systems, some variables, measured by sensors within close proximity to the drivetrain, are also incorporated. They are valuable additional indicators for deciding the degradation condition of the drivetrain and its components, and sometimes can provide incipient failure detection with superior performance (Feng, Qiu, Crabtree, Long and Tavner, 2011). Examples of these variables include temperature readings of the rotor, gearbox and generator, as well as the gearbox oil pressure.

A heuristic method is used to select SCADA variables based on variable name and measurement location. Given that SCADA data is typically recorded more frequently than CMS data in wind power applications, SCADA records are selected only when a retained CMS instance exists at matching time interval.

\subsection{CMS Data Feature Extraction}

For health assessment and diagnosis, values of SCADA variables can be directly used as health indicators, whereas for CMS data features are normally computed to reduce its dimension and obtain representative indicators. A toolbox of signal processing techniques for vibration-based wind turbine monitoring has been designed and developed (Siegel, Zhao, Lapira, AbuAli and Lee, 2013), to extract features corresponding with key drivetrain components such as bearings, shafts, and gears respectively.

\subsubsection{Time Domain Features}

Time domain features provide statistical measures of a variable. Three commonly used features for vibration analysis are root mean square (RMS), kurtosis and crest factor (Lebold, MacClintic, Campbell, Byington and Maynard, 2000). For a data vector $X_{i}, i=1,2,3 \ldots N$, these features are defined as:

$$
R M S=\sqrt{\frac{\sum_{i=1}^{N} X_{i}^{2}}{N}}
$$




$$
\begin{gathered}
\text { Kurtosis }=\frac{\sum_{i=1}^{N}\left(X_{i}-\bar{X}\right)^{4}}{N} /\left[\frac{\sum_{i=1}^{N}\left(X_{i}-\bar{X}\right)^{2}}{N}\right]^{2} \\
\text { Crest Factor }=\max \left(\left|X_{i}\right|\right) / R M S
\end{gathered}
$$

RMS is calculated as the vector's Euclidean norm, divided by the square root of vector length, as shown in Eq. (1). It represents the magnitude, or energy, of the vibration signal. A high RMS value can indicate indefinite or severe damages. Kurtosis, computed as Eq. (2), is the ratio between the vector's fourth moment about the mean and square of its second moment about the mean. It is essentially a measure of signal peakedness, which normally increases when damage causes impulses and unevenness in data. Crest factor can be used to detect high-amplitude impacts, when such impacts generate large signal impulse and increase the ratio between maximum value and RMS of the signal indicated by Eq. (3).

\subsubsection{Spectral Kurtosis Filtering}

To monitor damages in a complex mechanical system like a drivetrain, it is necessary to detect impulsive vibration behavior stimulated by defective gears or bearings. Time domain features of raw data can fulfill the task to a certain degree, but the impulsive behavior is often obscured by additive noise from irrelevant vibration resources. Therefore, band-pass filters in the frequency domain are applied to preserve the most impulsive frequency content and de-noise the signal.

Spectral kurtosis filtering (SKF) is a technique to optimize the configuration of band-pass filter for noise reduction. Based on time-frequency analysis results, it adopts the kurtosis computation from the time domain analysis to seek the most impulsive frequency band.

As developed by Antoni (2006) for non-stationary signal analysis, the short-time Fourier transform (STFT) of the signal is first calculated and denoted as $H(t, f)$. For each frequency index decided by the STFT, the kurtosis of its amplitude over discrete time is calculated as Eq. (4):

$$
S K_{X}(f)=\frac{\left\langle H^{4}(t, f)\right\rangle}{\left\langle H^{2}(t, f)\right\rangle^{2}}-2
$$

A statistical threshold $S_{\alpha}$ is computed (Antoni and Randall, 2006) to decide the significance of spectral kurtosis given level of significance $\alpha$ :

$$
S_{\alpha}=u_{1-\alpha} \frac{2}{\sqrt{K}}
$$

where $u_{1-\alpha}$ is the quantile with significance level $\alpha$, and $K$ is the number of time windows in STFT analysis. If the SK value for certain frequency is higher than the threshold, a
Wiener filter is multiplied with the frequency spectrum of the original signal, where the multiplier is square root of the SK value. Therefore, the frequency content that is originally impulsive with high SK level is further amplified, whereas the other content is attenuated. The signal is eventually transformed back to a time series for extracting time domain features.

An example for effect of spectral kurtosis filtering is shown in Figure 2, where filtered data (bottom plot in red) apparently accentuate impulsive behavior more than raw data (top plot in blue). To quantify the difference, time domain kurtosis values before and after the filtering are 5 and 20.4 respectively; crest factors are 6.1 and 16.3 respectively.
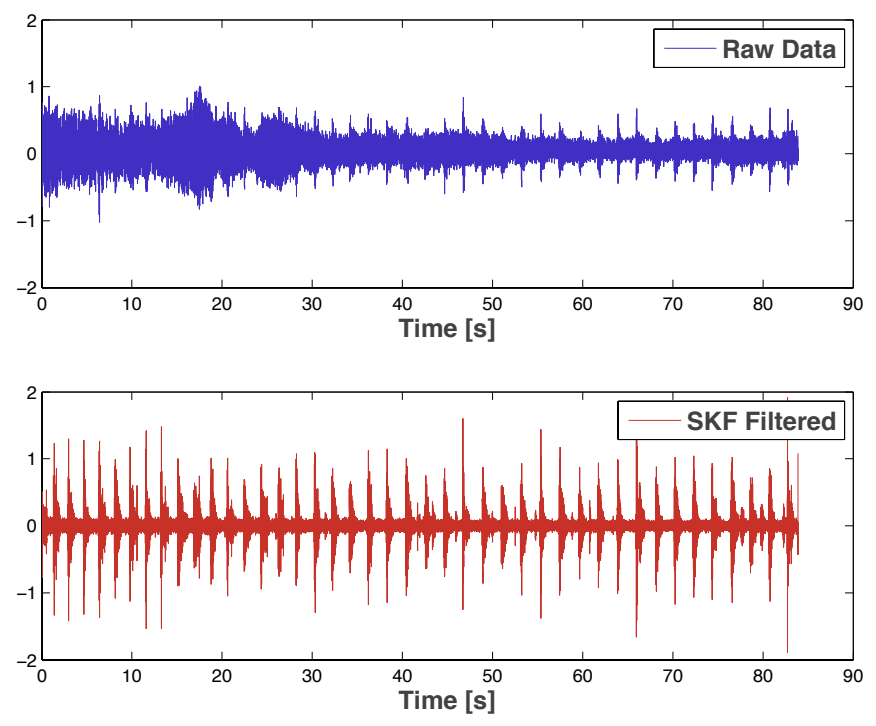

Figure 2. An example of spectral kurtosis filtering result

Spectral kurtosis filtering discovers the inherent dynamics of vibration spectrum, and automatically de-noises the signal without prior knowledge or visual inspection for band selection. In research with similar objectives, Barszcz and Randall (2009) previously investigated using spectral kurtosis for tooth crack detection in wind turbine planetary gears.

\subsubsection{Envelope Analysis}

As rolling element bearings and gear wheels rotate around their shafts in the gearbox system, bearing damages and gear defects often cause multiple impacts per each revolution and excite the resonant vibration of the entire structure. In vibration data, it results in an amplitude modulation phenomenon, where the structural resonance is the high-frequency carrier wave and the component fault frequency is the low-frequency envelope that modulates the waveform in time domain. 
In frequency domain, modulation behavior is represented with concentrated high-amplitude peaks around resonance frequency where fault frequencies exist as sidebands spaced on both sides of resonance. To extract signatures related with specific faults, demodulation is performed with following steps:

- A band-pass filter is designed to filter vibration data around the excited resonance frequency. The design could be achieved by modal analysis, observation of spectrum for collected vibration data, or aforementioned spectral kurtosis filtering technique.

- Envelope analysis of data that is processed with the band-pass filter. A frequently used method is Empirical Mode Decomposition (EMD), which is an iterative filtering process shown as Figure 3 (Peng, Tse and Chu, 2005).

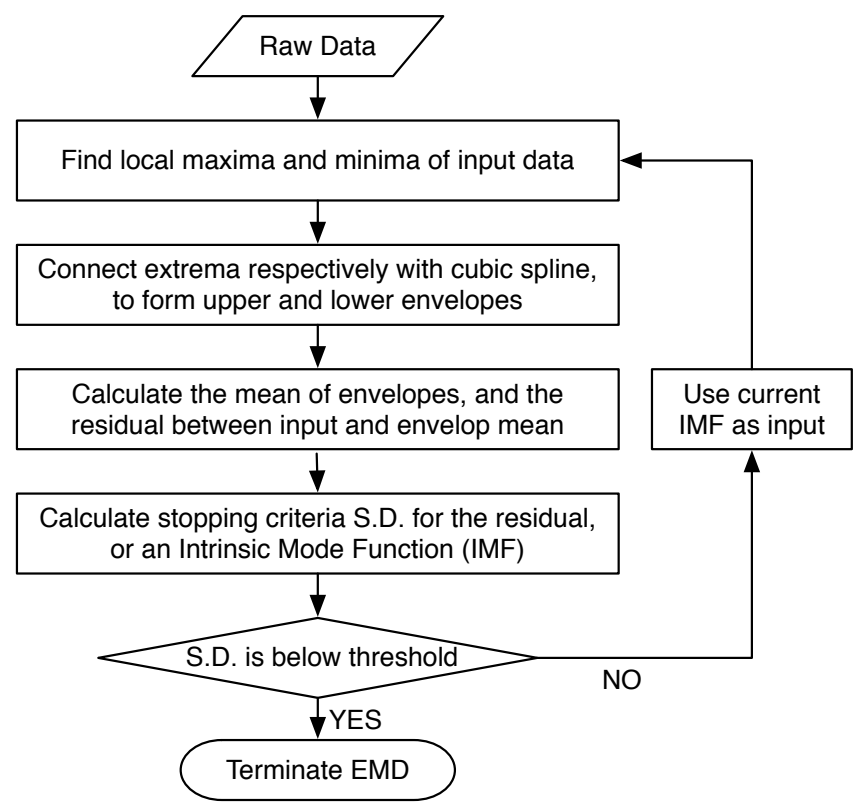

Figure 3. Empirical Mode Decomposition (EMD) process

- The method of extracting envelopes with local extrema has disadvantages of possible overshoots and breakpoints when a cubic spline is applied. To overcome such disadvantages, a Hilbert transform method is adopted (Liu, Riemenschneider and $\mathrm{Xu}$, $2006)$ to find the upper envelope of signal $x(t)$, by finding the principal value (PV) $y(t)$ with Eq. (6) first, and analytic signal $z(t)$ with Eq. (7). The envelope is eventually the absolute value of analytic signal $z(t)$.

$$
\begin{gathered}
y(t)=\frac{1}{\pi} P V \int_{-\infty}^{\infty} \frac{x(\tau)}{t-\tau} d \tau \\
z(t)=x(t)+i y(t)
\end{gathered}
$$

- After finding the envelope of band-pass filtered data with Hilbert transform, several feature extraction methods can be used to further analyze the envelope as signature of component defects. For example, bearing fault frequencies including ball pass frequency innerrace (BPFI), ball pass frequency outer-race (BPFO), ball fault frequency (BFF) and fundamental train frequency (FTF) for faults on bearing inner race, outer race, roller element and cage respectively. Furthermore, time domain statistics can also be indicators for defects, such as RMS and crest factor.

\subsubsection{Wavelet Energy Analysis}

The use of wavelets for time-frequency analysis as a method for automated feature extraction has seen a growing interest in the area of condition monitoring (Peng and Chu, 2004). For CMS vibration analysis, the focus is how to use wavelet analysis for feature extraction, and thus the discussion on the background of time-frequency analysis and continuous wavelet transform is omitted here. The wavelet decomposition and the wavelet packet decomposition are the more commonly used algorithms for feature extraction purposes, particularly in wind turbine monitoring area as well (Yang, Tavner and Wilkinson, 2008). The wavelet decomposition applies a filtering operation in which the signal is divided into an approximation signal (low frequency) and a detail signal (high frequency). The approximation signal consists of frequency content from 0 to approximately $1 / 4$ of the Nyquist frequency $\left(F_{n y q}\right)$, while the detail signal consists of frequency content from $1 / 4$ of the Nyquist frequency to $1 / 2$ of the Nyquist frequency. This represents the decomposition at the first level, and the approximation signal is further decomposed to a specified number of levels. The selection of the mother wavelet influences the filtering result, wherein higher coefficients and values can be obtained when the mother wavelet function is a closer match to the original signal (Jiang, Tang, Qin and Liu, 2011).

In general, the frequency content at level $n$ for the approximation signal is given by Eq. (8) and the frequency content for the detail signal at level $n$ is given by Eq. (9).

$$
\begin{gathered}
0 \leq f \leq \frac{F_{n y q}}{2^{n+1}} \\
\frac{F_{n y q}}{2^{n+1}} \leq f \leq \frac{F_{n y q}}{2^{n}}
\end{gathered}
$$




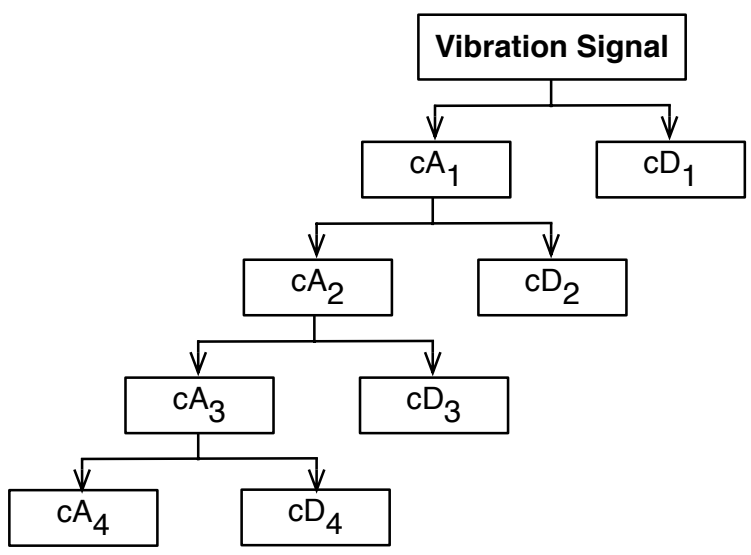

Figure 4. Wavelet Decomposition Diagram (Level 4)

An example wavelet decomposition diagram for level 4 is illustrated in Figure 4, where the approximation signal at each level is further decomposed. The wavelet decomposition only decomposes approximation signals, while the wavelet packet decomposition decomposes both approximation and detail signals.

Mechanical fault signatures for bearing, shaft, and gear components create amplitude and frequency modulation effects. In addition, these faults can excite the structural resonances of the system. Monitoring the vibration changes in different frequency bands from a baseline state is a way to monitor the overall health state of the drivetrain. The energy extracted at each node of the wavelet decomposition is used for monitoring the changes in the vibration in different frequency bands. The wavelet decomposition energy at each node can be calculated using Eq. (10), which consists of the squared summation of the coefficients at that particular node for the $N$ coefficients. The wavelet energy vibration feature is a normalized frequency band vibration value, in which the vibration at each node is normalized by the total energy in the vibration signal and the feature is given as a percentage value. An example calculation of the wavelet energy feature for the approximation signal for a level 4 decomposition is provided in Eq. (11). In this equation, the coefficients for the approximation signal are denoted as $w_{a 4}$, and the detail coefficients are denoted as $w_{d 1}, w_{d 2}, w_{d 3}$, and $w_{d 4}$ respectively. For this example of level 4 decomposition, 5 wavelet features would be extracted, since a feature would be extracted at each level for the detail signals, and a feature is extracted for the level 4 approximation signal.

$$
\begin{gathered}
\text { Energy } A_{4}=\sum_{i=1}^{N} w_{a 4 i}{ }^{2} \\
\frac{100 \times \sum w_{a 4}^{2}}{\sum w_{d 1}^{2}+\sum w_{d 2}^{2}+\sum w_{d 3}^{2}+\sum w_{d 4}^{2}+\sum w_{a 4}^{2}}
\end{gathered}
$$

\subsection{Drivetrain Overall Degradation Assessment}

Upon the completion of SCADA variable selection and CMS feature extraction, the set of features from all sensors and selected SCADA variables are used to evaluate drivetrain degradation as explained in subsequent sections of the paper. Degradation assessment estimates present drivetrain condition by comparing a feature distribution model with a known healthy condition as the baseline model. As operation conditions change for the wind turbine, the drivetrain work regime varies over time and affects its response even under comparable health condition. Therefore, the features are assumed to comprise distributions from multiple models, and a modeling method that can learn and represent data with a mixture model is preferred.

Moreover, a distance metric is used to quantify degradation by measuring the dissimilarity between present features and the baseline. A threshold can be defined as an unacceptable level for the distance, and fault detection can be confirmed when the distance measure exceeds the threshold.

In this study, a Self-organizing Maps (SOM) approach is used for degradation assessment (Kohonen, 1990). Being a type of artificial neural network, SOM is able to automatically discover signal patterns and organize signals to create spatial separation between clusters. When used for unsupervised learning tasks where the data labels are not available for classification, SOM can cluster data instances so that inter-cluster distance is high and intra-cluster distance is low.

To train a SOM, a 2D map is initialized with $m$ neurons corresponding with $n$ input vectors $x$ :

$$
x=\left[x_{1}, x_{2}, \ldots, x_{n}\right]^{T}
$$

Each neuron has a weight vector that has the same dimension $n$ of an input vector:

$$
w_{j}=\left[w_{j 1}, w_{j 2}, \ldots, w_{j n}\right]^{T}, j=1,2, \ldots, m
$$

For each of the input vectors, the Euclidean distance between the particular input vector and all weight vectors are calculated. The weight vector with smallest distance, hence highest similarity is chosen as the Best Matching Unit (BMU), $w_{c}$, for that input vector, as shown in Eq. (14).

$$
\left\|x_{i}-w_{c}\right\|=\min _{j}\left\{\left\|x_{i}-w_{j}\right\|\right\}
$$

After the first iteration of finding the BMUs, the values of weight vectors are updated so that each BMU is topologically closer to the input vector. The updated is computed as Eq. (15):

$$
w_{j}(t+1)=w_{j}(t)+\alpha(t) h_{j, w_{c}}(t)\left(x-w_{j}(t)\right)
$$


where $t$ denotes the iteration step; $h_{j, w_{c}}$ denotes the topological neighborhood kernel centered around the BMU, which is typically chosen as Gaussian function; and $\alpha(t)$ denotes the learning rate, which is monotonically decreasing with the training iteration. Through this competitive learning process where weight vectors that are closer to input space get updated with higher weight, the map of weight vectors eventually converge to a certain number of clusters.

Minimum quantization error (MQE) is the distance metric for SOM method ( $\mathrm{Yu}$ and Wang, 2009), computed as the Euclidean distance between an input vector and its BMU, as shown in Eq. (16). Therefore the training of SOM can be viewed as the process of minimizing the average MQE for input vectors and achieving the optimal map structure. For a testing process, where the present degradation condition is assessed, features are used as input vectors for the trained map as they are collected. The MQE value is calculated for each feature vector against its BMU in the trained SOM map, which can be found in the "codebook" of the SOM. The larger the MQE value is, the more severe the degradation is.

$$
M Q E=\left\|x-w_{B M U}\right\|
$$

\subsection{Fault Localization}

As drivetrain degradation grows and becomes significant, the MQE value is expected to exceed its prescribed threshold and trigger an alarm for fault. It is desirable to locate the fault at component level, so that specific advice can be provided for deciding which component is at a more critical condition and needs to be repaired.

With SOM-MQE technique being used, fault localization is achieved by computing MQE contribution of features and variables from each component. MQE is essentially the Euclidean norm of the vector subtraction between an input vector and its BMU, as shown in Eq. (17), where $e_{i}$ is the difference for the $i$ th feature among all $k$ features.

$$
M Q E=\sqrt{e_{1}^{2}+e_{2}^{2}+\cdots+e_{k}^{2}}
$$

The features can be grouped by drivetrain components based on their contextual information. CMS vibration features are grouped based on location of the sensor that generated the feature, whereas SCADA variables are grouped based on variable names. For each component, its contribution to MQE value is calculated as Eq. (18), where $e_{i}$ are features of the same component.

$$
\text { Contribution }=\frac{\sum e_{i}^{2}}{M Q E^{2}}
$$

\section{Case Study}

To further validate and implement the aforementioned methodology, a case study based on an offshore wind turbine is conducted.

\subsection{Description of Turbine and Data}

The test bed is a $3 \mathrm{MW}$ wind turbine. A split torque, threestage planetary gearbox is used to connect the rotor and the generator on the drivetrain. Schematics of the drivetrain, as well as locations of vibration sensors, are shown in Figure 5.

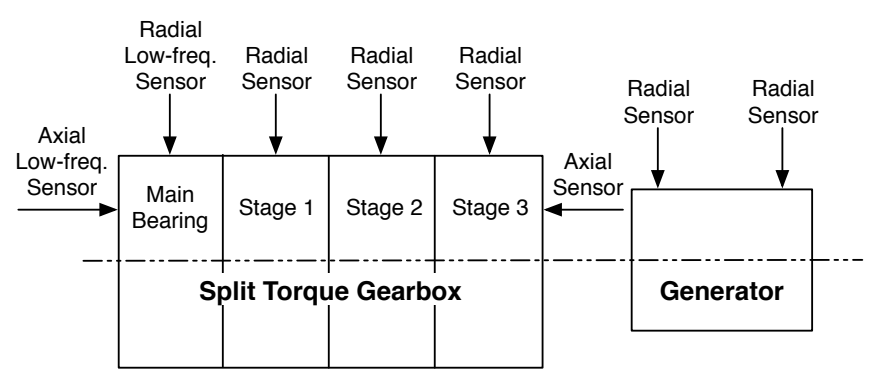

Figure 5. Schematics of test bed drivetrain

The first two stages each consist of a sun gear, planetary gears, a planet carrier and a ring gear, whereas the third stage is a parallel stage with a pair of gears and an output pinion. The input shaft of the gearbox drives ring gear of the first stage and planet carrier of the second stage simultaneously with identical rotation speed. For the first stage, planet carrier does not rotate, thus planetary gears only rotate on their own axes without rolling relative with its sun gear. The sun gear, driven by the ring gear through rotation of planetary gears, connects with the ring gear of the second stage. Therefore all of ring gear, planetary gears and planetary carrier rotate for the second stage, to drive the rotation of its sun gear. Then the sun gear of second stage connects with the third stage, and outputs rotation that drives the generator. Parameters of the gears are listed in Table 2, where CW means the rotation is clockwise and $\mathrm{CCW}$ means counterclockwise. The computation of overall gear ratio results in 76.64 , which is the nominal ratio between rotation speeds of generator and rotor shaft.

\begin{tabular}{l|l|l|l}
\hline Stage & Gear & No. Of Tooth & Rotation Direction \\
\hline \multirow{3}{*}{$1^{\text {st }}$} & Sun & 66 & CW \\
\cline { 2 - 4 } & Planet (8) & 37 & CW \\
\cline { 2 - 4 } & Ring & 142 & CCW \\
\hline \multirow{2}{*}{$2^{\text {nd }}$} & Sun & 30 & CCW \\
\cline { 2 - 4 } & Planet (4) & 62 & CCW \\
\cline { 2 - 4 } & Ring & 154 & CW \\
\hline \multirow{2}{*}{$3^{\text {rd }}$} & Input & 116 & CCW \\
\cline { 2 - 4 } & Pinion & 26 & CW \\
\hline
\end{tabular}

Table 2. List of known gear parameters

In total, eight (8) accelerometers are installed along the drivetrain (Figure 5), with two on the main bearing, four on 
the gearbox and two on generator. The sampling rate is 6250 $\mathrm{Hz}$ for all sensors.

The condition monitoring system is configured to collect vibration data from all accelerometers synchronously once per day at midnight, with a few exceptions that data is collected at a different time of the day or data is collected more than once per day. Data duration for all eight channels of each collected instance is around 85 seconds.

SCADA data is also available for the turbine unit, where statistics of mean, standard deviation, maximum and minimum for over a hundred variables are recorded every ten (10) minutes. The total duration of both CMS and SCADA data is fifteen (15) months. MATLAB ${ }^{\circledR}$ is used for developing all techniques described in previous Section and generating results for this case study.

\subsection{Analysis and Results}

A filtering algorithm (Grubbs, 1969) that detects outlier observations in a time series is used to reject drastic outliers for each SCADA variable in advance. In this algorithm, the null hypothesis is defined as there is no outlier in a distribution, whereas alternative hypothesis is defined as there is at lease one outlier in the distribution. For any given sample of the distribution, $X_{i}$, a Grubbs' test statistics $G$ is generated as Eq. (19), where $\bar{X}$ is the distribution's mean value and $\sigma$ is the distribution's standard deviation.

$$
G=\left|\bar{X}-X_{i}\right| / \sigma
$$

A critical value $Z$ is computed as shown in Eq. (20), where $N$ is distribution sample size and $t$ is critical value of the tdistribution with $N-2$ degrees of freedom and $\alpha /(2 N)$ of significance level. If $G>Z, X_{i}$ is determined to be an outlier and null hypothesis is rejected.

$$
Z=\frac{N-1}{\sqrt{N}} \sqrt{\frac{t^{2}}{N-2+t^{2}}}
$$

For each SCADA variable, its maximum and minimum values are tested with a significance level of 0.05 , and rejected if they are determined to be outliers. Extrema values of the filtered distribution will be tested repeatedly until no outlier is detected.

SCADA records with apparent timestamp error are rejected as well.

For each available CMS data instance, a SCADA record with a matching timestamp is selected as the reference for deciding whether the CMS instance should be discarded or kept. In the case when there is no SCADA record with exact same timestamp for a particular CMS instance since data sampling between the two systems may not be synchronized in most instances, the first SCADA record sampled right after the CMS instance is chosen as the reference.
Four variables in reference SCADA records are then examined with following rules:

- $\quad$ Rotor speed average [rpm] is higher than 0 ;

- Generator speed average $[\mathrm{rpm}]$ is higher than 0;

- Average active power $[\mathrm{kw}]$ is higher than 0 ;

- Average wind speed $[\mathrm{m} / \mathrm{s}]$ is higher than cut-in wind speed, which in this case is 2.

The corresponding CMS instance is retained when the drivetrain is operational, which is indicated, in most cases, by all four aforementioned rules being met.

Reference SCADA records of retained CMS instances are kept for future analysis. In these records, four variables are selected for degradation assessment:

- $\quad$ Rotor bearing temperature average;

- Gearbox stage 1 temperature average;

- Gearbox stage 2 temperature average;

- Gearbox stage 3 temperature average.

The selected temperature readings are then subtracted by the variable Environment temperature average, to offset the seasonal effect on the absolute reading of the variables.

For feature extraction of each channel of CMS vibration data, four categories of features are extracted:

- RMS, kurtosis and crest factor are time domain features;

- Spectral kurtosis is used to filter vibration waveform, with a beforehand STFT of window size 256 samples and 80 percent of overlap. RMS, peak-to-peak and kurtosis values are extracted from the filtered time domain signal;

- Envelope analysis is used to demodulate the signal around resonance frequencies, where resonance frequency is often found at the interval between 1000 $\mathrm{Hz}$ and $1600 \mathrm{~Hz}$. Five features are extracted from the demodulated signal: RMS of envelope, RMS of bandpass filtered data, maximum peak of envelope spectrum in low frequency range, frequency index of the peak, and crest factor of envelope spectrum. In this specific case study, bearing configuration parameters that are indispensable for bearing fault frequency calculation are not available. Therefore fault frequencies are not considered in this example.

- Wavelet energy analysis is conducted with Daubechies 4 wavelet, and five features, including four energy bands for four levels of detail signal and one energy band for level 4 approximation signal, are extracted.

In this case study, features are extracted only from sensor 1 to sensor 6 since the gearbox system is of higher interest and it is more suitable to study analytical methods that are specific for the generator independently. 
In total, 96 CMS features are extracted with 16 features per each accelerometer. With selected SCADA variables, 100 health indicators are included in SOM-MQE calculation for degradation assessment. For this case study, a Monte Carlo based statistical method is (Bechhoefer, He \& Dempsey, 2011) adopted to generate a threshold for triggering fault detection when MQE value exceeds the threshold. To be conservative and avoid users' disbelief due to false alarms, a probability of false alarm (PFA) is set as $10^{-6}$. Gaussian distribution and Rayleigh distribution are fit with the first 30 MQE instances, where Rayleigh distribution has a lower negative $\log$ likelihood value and is a better fit for the instances. The same test is conducted for varying number of instances (25 - 35) and Rayleigh distribution consistently outperforms Gaussian distribution, therefore the distribution is assumed to be Rayleigh in this case. Threshold values are calculated based on the different numbers of training instances, and the average is taken to be the eventual threshold.

The reminder of the historical data is used for testing the SOM model. Each instance is input to the trained SOM, to generate a MQE value. Eventually, the trend of MQE (in $\mathrm{dB})$ progression over time is shown in Figure 6.
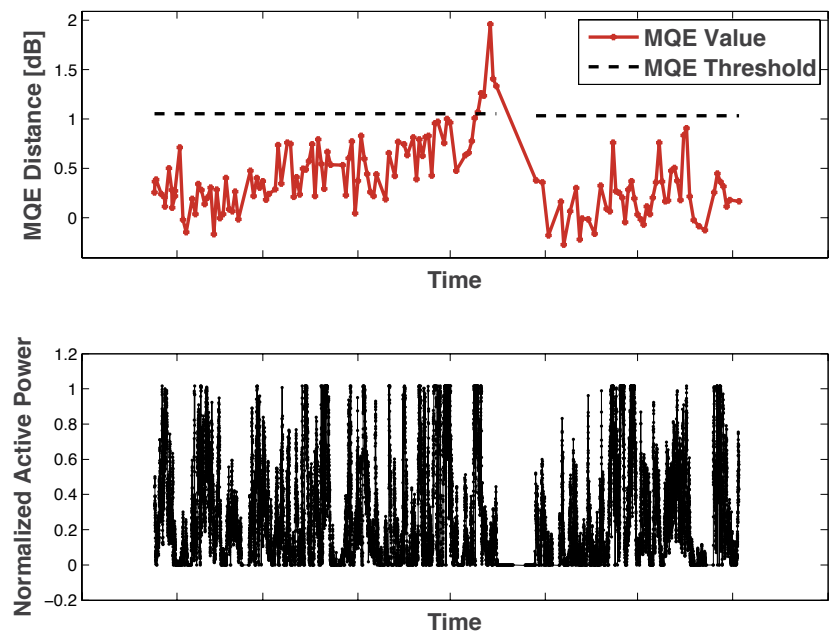

Figure 6. SOM-MQE result for degradation assessment

As observed from the SOM-MQE result, there is a short duration in the middle of the history when MQE value noticeably exceeded the MQE threshold. Due to data confidentiality issue, the exact dates cannot be revealed. However, it can be found from SCADA variable Average active power $[\mathrm{kW}]$ that the turbine unit was producing zero power for a two-week duration. It was probably triggered by simply monitoring the level of SCADA variables and pausing the turbine operation due to certain alerts.

In comparison, the MQE excess occurred about five days before the operation pause. The result shows that SOMMQE is capable of detecting drivetrain anomaly at an early stage.
After the wind turbine resumes operation, the SOM model is re-trained since there might have been component replacement and drivetrain behavior should be compared with a new baseline. As shown in Figure 6, the new SOM model has a new MQE threshold for fault detection as well.

For fault localization, features are grouped based on sensor locations in the schematics and SCADA variable names. The components here are denoted as component 1, 2, 3 and 4 , where actual component names are omitted as proprietary information. When MQE exceeds its threshold and fault is detected, the contribution of MQE increase is calculated for each component based on Eq. (18), which results in 0.9, $0.05,0.02$ and 0.03 for the occurrence of the major downtime. Therefore the critical component in this case is decided to be component 1 .

A radar chart is created to view component criticality simultaneously (Figure 7). In this chart, each axis represents the contribution of each component to MQE abnormality. The closer the data point is to the center, the smaller the contribution is.

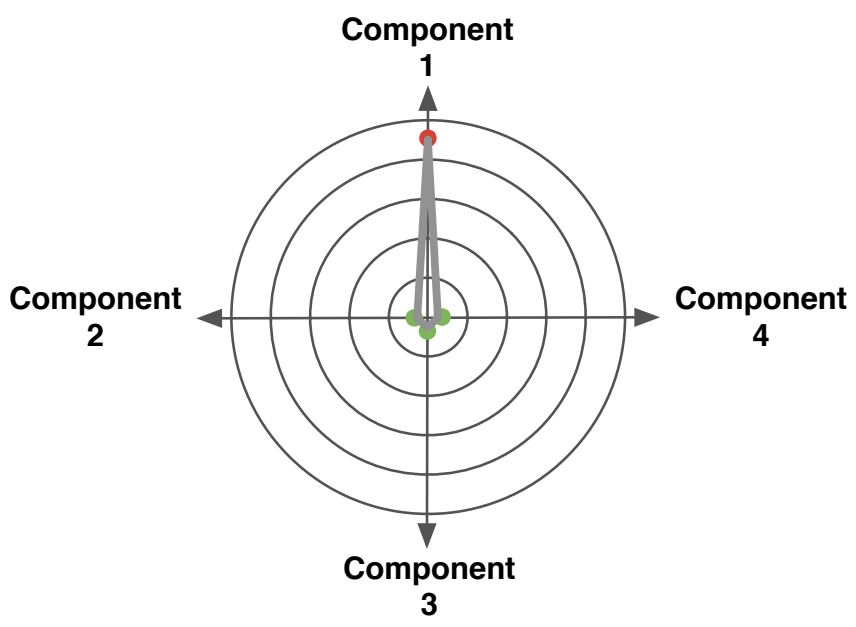

Figure 7. Radar chart for fault localization

\subsection{Visualization}

To apply the developed tools for large-scale wind farms, a monitoring platform prototype is established for data management, visualization, analysis and fault reporting. The software modules include a) main interface, which directs user to different analytical modules: b) data organization, which sorts data instances and convert them to a compatible format; c) signal visualization and filtering, which provides visual observation of raw signal and configuration of data filtering; d) feature extraction, which enables feature configuration and extraction for various feature types, as well as SCADA variable selection; and e) degradation assessment and fault localization. The main module is able to invoke any other modules for particular tasks, and results from a former module are archived so that a latter module 
can use them as input. When the analytical methods are improved and validated with more turbine units and data, the platform can operate in a systematic manner to analyze raw SCADA and CMS data and to provide users with direct health degradation information.

\section{CONCLUSiOn AND FUtURe WORK}

In this paper, an integrated methodology of degradation assessment and fault localization for wind turbine drivetrain components is presented. The result of the methodology is achieved by combining input from SCADA system and $\mathrm{CMS}$, and validated with a planetary gearbox system of an offshore wind turbine.

Besides using selected SCADA variables, a few feature extraction methods are employed to extract health indicators from CMS vibration data. The methods include time domain features, spectral kurtosis filtering, envelope analysis and wavelet energy analysis. A Self-organizing Map and minimum quantization error approach is adopted to evaluate the degradation condition of drivetrain, and contribution calculation is used to decide the location of defect on the drivetrain. In the case study, an incipient defect is detected and located before detection by the existing system, indicating the potential of predictive monitoring with the presented methods.

In terms of future work, there are a few items to be considered for improving the methods and applications

- Regarding wavelet transform for feature extraction, the selection of mother wavelet function is crucial for obtaining the optimal decomposition results. Rather than depending on experience, preference or visual inspection, an intelligent method can be designed and validated for monitoring either onshore or offshore turbine drivetrains. In literature, there has been interesting and valuable investigation for reference (Rafiee, Tse, Harifi and Sadeghi, 2009).

- There are other multimodal methods that are applicable for degradation assessment. For example, Gaussian mixture model (GMM) estimates data distribution as linear combination of multivariate Gaussian distribution components. A L2 distance metric can be used to measure degradation.

- In the case study, rotation speed for CMS vibration data is missing. As a result, there are some techniques that could not be evaluated on the test bed, such as time synchronous average, especially for the variable speed transmission system of wind turbines (Zhang, Wen and $\mathrm{Wu}, 2012$ ). If rotational speed can be made available in the future, perhaps through a tachometer signal, more in-depth analysis can be conducted to investigate fault diagnosis methods.

- As bearing specifications are not available for the presented case study, bearing failure frequencies are not inspected and potential bearing faults are not explored. Adding bearing-specific knowledge in the future will enable bearing diagnosis for different stages of drivetrain with given sensors.

- The CMS instance selection method, which is proposed and implemented in this paper, can be incorporated with control of data sampling for CMS. CMS can refer to SCADA variables to evaluate if certain time duration is suitable for vibration data acquisition. An adaptive sampling mechanism can be developed to ensure CMS data quality, improve computation efficiency, and enhance degradation model accuracy.

\section{ACKNOWLEDGEMENT}

The authors would like to acknowledge the collaborators at the Sinovel Wind Group, especially Mr. Wei Hu, Mr. Feng Wang and Dr. Yubin Zhu, for their support in terms of condition monitoring system expertise, wind turbine operation experience and providing data for the case study. The authors would like to acknowledge the National Science Foundation (NSF) for its support of research and project activities at Industry/University Cooperative Research Center for Intelligent Maintenance Systems (IMS) at the University of Cincinnati, as well as IMS industry members for offering applied research opportunities that proves to be fundamental to this specific area. The authors would like to acknowledge Ms. Christina Lucas for reviewing the manuscript.

\section{APPENDIX}

Screenshots of software modules discussed in Section 3.3 are included in this appendix.

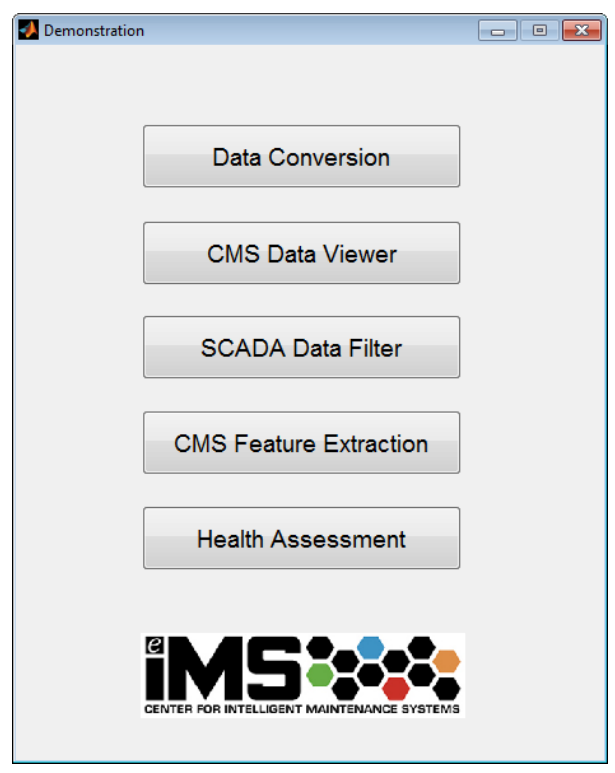

a) Main interface 


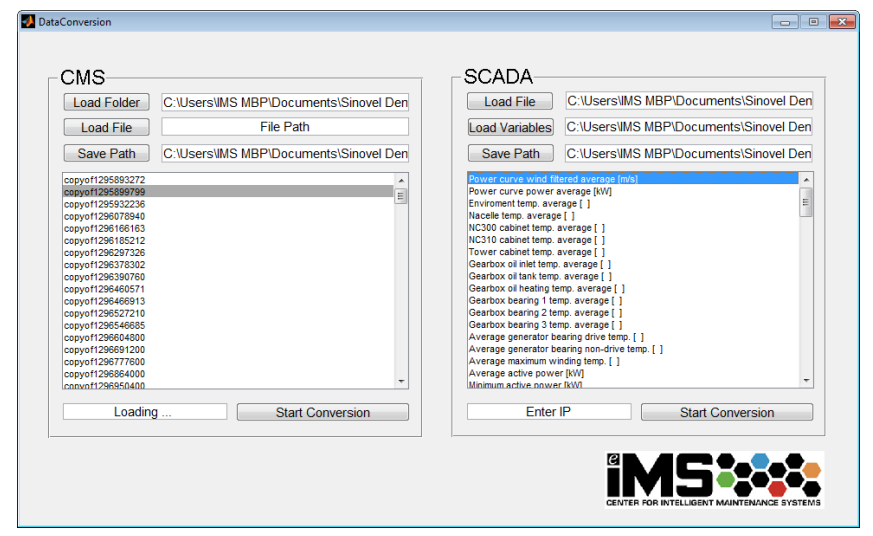

b) Data organization module

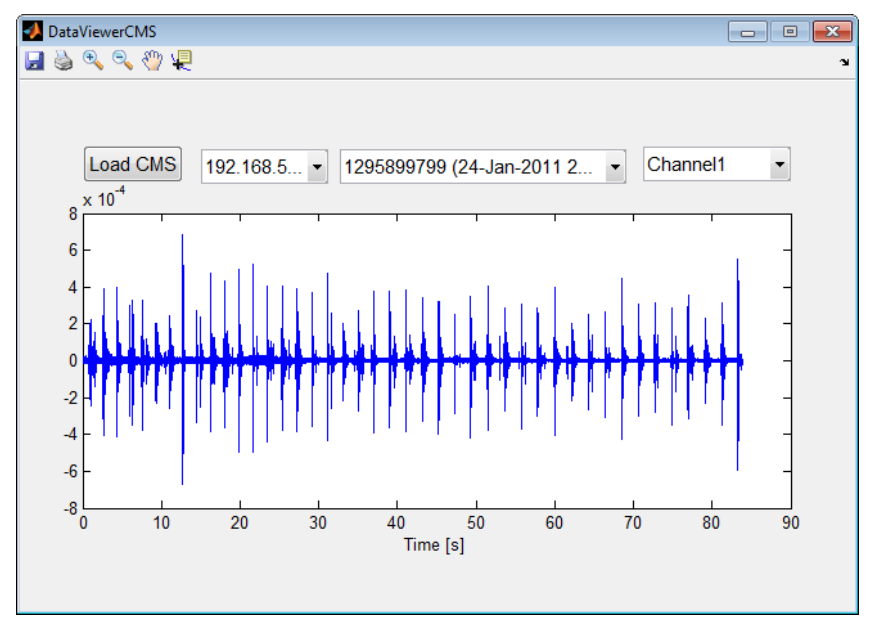

c) CMS data visualization module

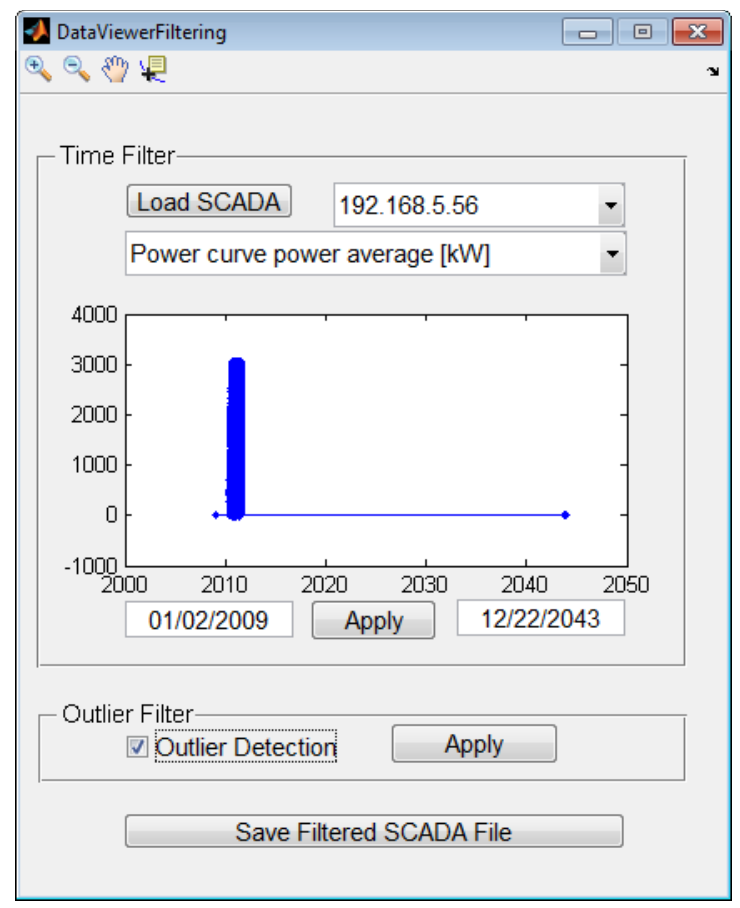

d) SCADA variable filtering module

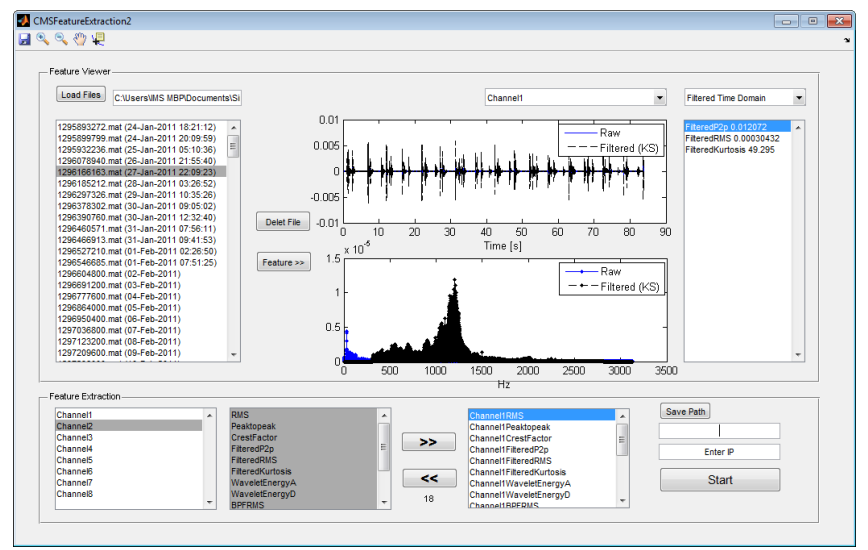

e) CMS feature extraction module 


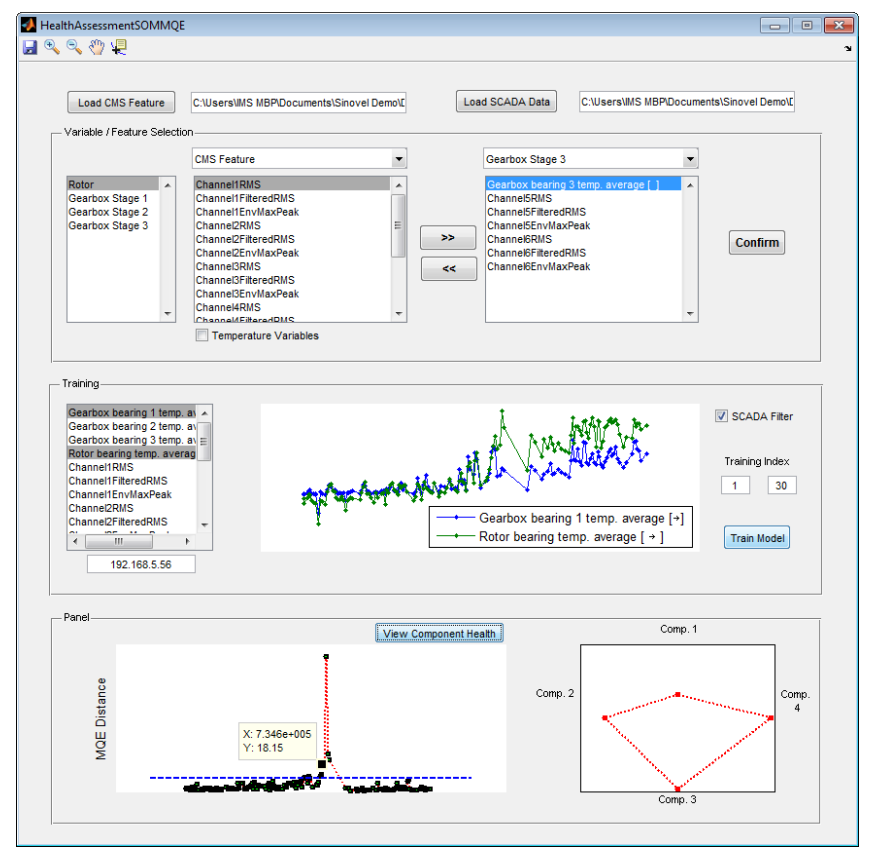

f) Degradation assessment and fault localization module

Figure 8. Prototype of wind farm monitoring platform

\section{REFERENCES}

Amirat, Y., Benbouzid, M. E. H., Al-Ahmar, E., Bensaker, B., \& Turri, S. (2009). A brief status on condition monitoring and fault diagnosis in wind energy conversion systems. Renewable and Sustainable Energy Reviews, $13 \quad$ (9), pp. 2629-2636. doi:10.1016/j.rser.2009.06.031

Antoni, J. (2006). The spectral kurtosis: a useful tool for characterising non-stationary signals. Mechanical Sysetms and Signal Processing, 20 (2), pp. 282-307. doi:10.1016/j.ymssp.2004.09.001

Barszcz, T. \& Randall, R. (2009). Application of spectral kurtosis for detection of a tooth crack in the planetary gear of a wind turbine. Mechanical Systems and Signal Processing, $23 \quad$ (4), pp. 1352-1365. doi:10.1015/j.ymssp.2008.07.019

Bechhoefer, E., He, D., \& Dempsey, P. (2011). Gear health threshold setting based on a probability of false alarm. Annual Conference of the Prognostics and Health Management Society, September 25 - 20, Montreal, Canada.

Crabtree, C. J., Feng, Y., \& Tavner, P. J. (2010). Detecting incipient wind turbine gearbox failure: A signal analysis method for on-line condition monitoring. Scientific Track Proceedings of European Wind Energy Conference, April 20-23, Warsaw, Poland.

Edwin, W., Theo, V., Henk, B., Luc, R., Xiang, J., \& Simon, W. (2008). Assessment of condition monitoring techniques for offshore wind farms. Journal of Solar Energy Engineering, 130 (3), pp. 0310041-0310049.
Entezami, M. (2010). Wind turbine condition monitoring system. Doctoral dissertation report. University of Birmingham, Birmingham, United Kindom. http://www.sampaolesi-

impiantielettrici.com/documenti/eolico/Wind_Turbines _Condition_Monitoring_Systems_University_of_Birmi ngham_2010.pdf

Feng, Y., Qiu, Y., Crabtree, C. J., Long, H., \& Tavner, P. J. (2011). Use of SCADA and CMS signals for failure detection and diagnosis of a wind turbine gearbox. European Wind Energy Conference \& Exhibition, March 14-17, Brussels, Belgium.

Fraunhofer-Institute for Wind Energy and Energy System Technology. (2005). Final report: Advanced maintenance and repair for offshore wind farms using fault prediction and condition monitoring techniques. http://ec.europa.eu/energy/renewables/wind_energy/doc /offshore.pdf

Global Wind Energy Council. (2012). Global Wind Energy Outlook 2012. http://www.gwec.net/wpcontent/uploads/2012/11/GWEO_2012_lowRes.pdf

Grubbs, F. E. (1969). Procedures for detecting outlying observations in samples. Technometrics. 11 (1), pp. 121. doi:10.1080/00401706.1969.10490657

Hameed, Z., Hong, Y. S., Cho, Y. M., Ahn, S. H., \& Song, C. K. (2009). Condition monitoring and fault detection of wind turbines and related algorithms: a review. Renewable and Sustainable Energy Reviews, 13 (1), pp. 1-39. doi:10.1016/j.rser.2007.05.008

Jiang, Y., Tang, B., Qin, Y., \& Liu, W. (2011). Feature extraction method of wind turbine based on adaptive Morlet wavelet and SVD. Renewable Energy, 36 (8), pp. 2146-2153. doi:10.1016/j.renene.2011.01.009

Kohonen, T. (1990). The self-organizing map. Proceedings of the IEEE, 78 (9), pp. 1464-1480.

Lapira, E., Siegel, D., Zhao, W., Brisset, D., Su, J., Wang, C., AbuAli, M., \& Lee, J. (2011). A systematic framework for wind turbine health assessment under dynamic operating conditions. Proceedings of the $24^{\text {th }}$ International Congress on Condition Monitoring and Diagnostics Engineering Management, (1-9), May 30 June 1, Stavanger, Norway.

LeBlanc, M., \& Graves, A. (2011). Condition monitoring systems: trends and cost benefits. NREL Wind Turbine Condition Monitoring Workshop, September 19-20, Broomfield,

CO. http://www.nrel.gov/wind/pdfs/day1_sessioni_04_garra dhassan_leblanc.pdf

Lebold, M., McClintic, K., Campbell, R., Byington, C., \& Maynard, K. (2000). Review of vibration analysis methods for gearbox diagnostics and prognostics. Proceedings of the $54^{\text {th }}$ Meeting of the Society for Machinery Failure Prevention Technology (623-634), May 1-4, Virginia Beach, VA, USA

Liu, B., Riemenschneider, S., \& Xu, Y. (2006). Gearbox fault diagnosis using empirical mode decomposition 
and Hilbert spectrum. Mechanical Systems and Signal Processing, $20 \quad$ (3), pp. 718-734. doi:10.1016/j.ymssp.2005.02.003

Lu, B., Li, Y., Wu, X., \& Yang, Z. (2009). A review of recent advances in wind turbine condition monitoring and fault diagnosis. Proceedings of IEEE Power Electronics and Machines in Wind Applications, (1-7), June 24-26, Lincoln, NE.

McMillan, D., \& Ault, G. W. (2007). Quantification of condition monitoring benefit for offshore wind turbines. Wind Engineering, 31 (4), pp. 267-285. doi: $10.1260 / 030952407783123060$

Meadows, B. (2011). Offshore wind O\&M challenges. NREL Wind Turbine Condition Monitoring Workshop, September 19-20, Broomfield, CO. http://www.nrel.gov/wind/pdfs/day1_sessioni_05_nrel_ meadows.pdf

Musial, W., \& Ram, B. (2010). Large-scale offshore wind power in the United States: Assessment of opportunities and barriers. National Renewable Energy Laboratory Report No. TP-500-40745.

Peng, Z. K., \& Chu, F. L. (2004) Application of the wavelet transform in machine condition monitoring and fault diagnostics: a review with bibliography. Mechanical Systems and Signal Processing, 18 (2), pp. 199-221. doi:10.1016/S0888-3270(03)00075-X

Peng, Z. K., Tse, P. W., \& Chu, F. L. (2005). An improved Hilbert-Huang transform and its application in vibration signal analysis. Journal of Sound and Vibration, 286 (12), pp. 187-205. doi: 10.1016/j.jsv.2004.10.005

Qiu, Y., Feng, Y., Tavner, P., Richardson, P., Erdos, G., \& Chen, B. (2012). Wind turbine SCADA alarm analysis for improving reliability. Wind Energy, 15 (8), pp. 951966. doi: 10.1002/we.513

Rafiee, J., Tse, P. W., Harifi, A., \& Sadeghi, M. H. (2009). A novel technique for selecting mother wavelet function using an intelligent fault diagnosis system. Expert Systems with Applications, 36 (3), pp. 48624875. doi:10.1016/j.eswa.2008.05.052

Sheng, S., \& Veers, P. (2011). Wind turbine drivetrain condition monitoring - An overview. Machinery Failure Prevention Technology: The Applied Systems Health Management Conference, May 10-12, Virginia Beach, VA.

Siegel, D., Zhao, W., Lapira, E., AbuAli, M., \& Lee, J. (2013). A comparative study on vibration-based condition monitoring algorithms for wind turbine drive trains. Wind Energy, doi:10.1002/we.1585

Yang, W., Tavner, P. J., \& Wilkinson, M. R. (2008). Condition monitoring and fault diagnosis of a wind turbine synchronous generator drive train. IET Renewable Power Generation, 3 (1), pp. 1-11. doi: 10.1049/iet-rpg:20080006

Yu, J., \& Wang, S. (2009). Using minimum quantization error chart for the monitoring of process states in multivariate manufacturing processes. Computers \&
Industrial Engineering, 57 (4), pp. 1300-1312. doi:10.1016/j.cie.2009.06.009

Zhang, X., Wen, G., \& Wu, T. (2012). A new time synchronous average method for variable speed operating condition gearbox. Journal of Vibroengineering, 14 (4), pp. 1766-1774.

\section{BIOGRAPHIES}

Wenyu Zhao is currently a Ph.D. researcher at Center for Intelligent Maintenance Systems (IMS) at the University of Cincinnati, and a graduate student with the School of Dynamic Systems. He has conducted research and industry projects in area of Prognostics and Health Management (PHM) for areas including rotary machinery, renewable energies, hydraulic system and vehicle system. Prior to joining IMS, he received his B.S.E in Mechanical Engineering from Shanghai Jiao Tong University (SJTU). His work experience includes internships at Parker Hannifin Corp. and Metal Industry Research and Development Centre. He won $2^{\text {nd }}$ place in 2009 PHM Data Challenge. His current research interest is to develop a unified framework and methodology for prognostics of an interconnected system.

David Siegel is currently a Ph.D. student in Mechanical Engineering at the University of Cincinnati, and a research assistant for the Center for Intelligent Maintenance Systems. Related work experience in the field of prognostics and health management include internships at General Electric Aviation, and at the U.S Army Research Lab. He is also a two-time winner of the prognostics and health management data challenge. His current research focus is on componentlevel prognostic methods, as well as health monitoring algorithms for systems operating under multiple operating regimes.

Jay Lee is an Ohio Eminent Scholar and L.W. Scott Alter Chair Professor in Advanced Manufacturing at the University of Cincinnati. He is the founding director of National Science Foundation (NSF) Industry/University Cooperative Research Center (I/UCRC) for Intelligent Maintenance Systems (IMS), which is a multi-campus NSF Center of Excellence between the University of Cincinnati (lead institution), the University of Michigan, and the Missouri University of Science and Technology. His current research areas include autonomic computing, embedded IT and smart prognostics technologies for industrial and healthcare systems, cloud-based prognostics as a service, self-aware sensor and system, and dominant design tools for product and service innovation. He is a Fellow of ASME, SME, and International Society of Engineering Asset Management (ISEAM). 